\title{
Mortalidad a cinco años de la fijación interna versus artroplastia total en pacientes de edad avanzada con fractura de cadera. Metaanálisis
}

\author{
5-years mortality of internal fixation vs total hip arthroplasty \\ in elderly patients with hip fracture. A meta-analysis
}

\author{
Paola Maritza Zamora-Muñoz, ${ }^{\star}$ Juan Francisco Molina-López, ${ }^{\ddagger}$ Ángel Cruz-Miranda, ${ }^{\S}$ Rodolfo Rivas-Ruiz
}

Citar como: Zamora-Muñoz PM, Molina-López JF, Cruz-Miranda Á, Rivas-Ruiz R. Mortalidad a cinco años de la fijación interna versus artroplastia total en pacientes de edad avanzada con fractura de cadera. Metaanálisis. An Med ABC. 2021; 66 (2): 86-90. https://dx.doi. org/10.35366/100475

\section{RESUMEN}

Introducción: La reducción abierta y fijación interna, así como la artroplastia total de cadera son las dos técnicas quirúrgicas más empleadas en fracturas desplazadas de cuello femoral en pacientes de edad avanzada. Sin embargo, existe controversia en cuanto a la mortalidad a largo plazo entre ambos métodos de tratamiento. Objetivo: Determinar el tratamiento con menor mortalidad a cinco años en pacientes de edad avanzada con fractura de cadera. Material y métodos: Se realizó una búsqueda electrónica utilizando las bases de PubMed (MEDLINE) de todos los estudios publicados hasta agosto de 2020. Se incluyeron en el análisis siete ensayos clínicos aleatorizados que comparan la reducción abierta y fijación interna vs artroplastia total de cadera. El metaanálisis se llevó a cabo utilizando el programa RevMan 5.4 de la colaboración Cochrane. La heterogeneidad entre los estudios se evaluó con I-cuadrada $\left(\mathrm{I}^{2}\right)$ y se realizaron los cálculos y gráficas de resultados con forest plot y funnel plot para evaluar el sesgo de publicación. Resultados: Se encontró menor tasa de mortalidad a cinco años en artroplastia total de cadera vs

\section{ABSTRACT}

Introduction: Open reduction and internal fixation and total hip arthroplasty are the common techniques of femoral neck fractures treatment in elderly patients. Nevertheless, there is a controversy about long term mortality between both treatment modality. The purpose of the present study is to determine which treatment has lower long term mortality at five years in elderly patients with hip fracture. Material and methods: We perform an electronic search at PubMed (MEDLINE) database of papers published until August 2020. Seven clinical trials that compare internal fixation vs hip arthroplasty were included. For meta-analysis the RevMan 5.4 form Cochrane collaboration was used. The heterogenicity between studies were evaluated with $I$-squared $\left(I^{2}\right)$ and the graphics and calculus were performed with forest plot and funnel plot for bias publication. Results: There is a less mortality at five years for THA vs ORIF (45.2\% vs $49.3 \% ; R R=0.82 ; C I 95 \%$ 0.68-0.98). The heterogenicity was $I^{2}=65 \%(p<0.05)$, so an aleatory analysis method was performed. The funnel plot analysis showed a bias publication in two studies toward more mortality risk in THA. Conclusions:
* Maestra en Ciencias Médicas. Departamento de Ortopedia y Traumatología del Centro Médico ABC Campus Santa Fe. Ciudad de México, México.

‡ Maestro en Ciencias Médicas. Departamento de Cirugía General del Centro Médico ABC Campus Santa Fe. Ciudad de México, México.

$\S$ Departamento de Ortopedia y Traumatología del Centro Médico ABC. Ciudad de México, México.

In Coordinación de Investigación en Salud. Centro de Adiestramiento e Investigación Clínica. Ciudad de México, México.

Recibido: 03/04/2021. Aceptado: 03/06/2021.
Correspondencia:

Paola Maritza Zamora-Muñoz

E-mail: dra.paolazamora@me.com

Abreviaturas:

RAFI = Reducción abierta y fijación interna.

ATC $=$ Artroplastia total de cadera.

$E C A=$ Ensayos clínicos aleatorizados . 
reducción abierta y fijación interna $(45.2 \%$ vs $49.3 \%$; $\mathrm{RR}=$ 0.82 ; IC 95\% 0.68-0.98). La heterogeneidad obtenida fue de $\mathrm{I}^{2}$ $=65 \%(\mathrm{p}<0.05)$, por lo que se realizó análisis por métodos aleatorios. Se hizo un análisis de funnel plot, donde se identificó un sesgo de publicación de dos estudios que confieren mayor riesgo de mortalidad al grupo de artroplastia total de cadera. Conclusiones: Con base en los resultados, se encontró que la artroplastia total de cadera presenta menor tasa de mortalidad a cinco años vs reducción abierta y fijación interna.

Palabras clave: Fractura de cadera, reducción abierta y fijación interna, artroplastia total de cadera, metaanálisis.

Nivel de evidencia: III
Total hip arthroplasty has lower mortality rate at five years compared with internal fixation.

Keywords: Hip fracture, open reduction and internal fixation, total hip arthroplasty, meta-analysis.

Level of evidence: III

cipantes fueran individuos mayores de 65 años con diagnóstico de fractura no desplazada de cuello femoral. Los artículos deben incluir la comparación de los dos distintos tipos de intervención: ATC vs RAFI. El desenlace buscado fue mortalidad a cinco años.

\section{Criterios de selección}

Para la inclusión de los resultados se consideraron únicamente ensayos clínicos aleatorizados (ECA) en idioma inglés. Los estudios incluidos cumplieron con los siguientes criterios de selección: 1) pacientes de edad avanzada (mayores de 65 años) con fractura desplazada del cuello femoral, 2) comparación con las intervenciones fijación interna (RAFI) vs la artroplastia total de cadera (ATC), 3) reportar mortalidad a cinco años.

\section{Evaluación del riesgo de sesgos en los estudios incluidos}

Ambos investigadores revisaron de forma independiente la metodología de cada uno de los artículos. Los principales sesgos evaluados fueron: sesgo de selección, ejecución, detección, reporte y tipo de cegamiento (Tabla 1).

\section{RESULTADOS}

Se encontraron 21 ensayos clínicos aleatorizados que comparan RAFI vs ATC. Se excluyeron 14 de los 21 ECA debido a que no cumplían con los criterios de inclusión. Seis de los 14 estudios descartados no tenían concordancia en una de las técnicas quirúrgicas con las maniobras de interés para el estudio, siete estudios más no reportaban el objetivo primario y un estudio fue un análisis de costos, por lo que fueron descartados (Figura 1).
Para esta revisión se incluyeron sólo estudios de tipo ensayo clínico aleatorizado (ECA), donde los parti-

Se realizó una búsqueda electrónica utilizando las to de 2020) con artículos publicados sólo en idioma inglés. Se utilizaron las palabras clave: internal fixation, total hip arthroplasty, hip fracture y elderly en combinación con operadores booleanos $A N D / O R$.

\section{Criterios de selección}


Tabla 1: Descripción de riesgo de sesgo de Bachrach y colaboradores y Cao y su equipo.

\begin{tabular}{lccc}
\hline Sesgo & Autor, año & Riesgo & Comentario \\
\hline Generación en la aleatorización (sesgo de selección & Bachrach, 2000 & Alto & La aleatorización fue realizada por el cirujano \\
& Cao, 2014 & Bajo realizó con sobre cerrado & No especifica el método \\
Encubrimiento de la asignación (sesgo de selección) & Bachrach, 2000 & No describe & Se aclara la forma de asignación \\
& Cao, 2014 & Bajo & Doble ciego \\
Cegamiento de participantes (sesgo de ejecución) & Bachrach, 2000 & Bajo & Bajo ciego \\
& Cao, 2014 & Bajo & Doble ciego \\
Cegamiento de resultado (sesgo de detección) & Bachrach, 2000 & Bajo & Siple ciego \\
& Cao, 2014 & Bajo & Seguimiento a 5 años \\
Información de resultados incompleta (sesgo de deserción) & Bachrach, 2000 5 años \\
& Cao, 2014 & Bajo & Análisis por intención a tratar \\
Reporte selectivo de resultados (sesgo de reporte) & Bachrach, 2000 & Análisis por intención a tratar \\
Otros sesgos & Cao, 2014 & - & -
\end{tabular}

\section{Resultado principal}

Se incluyó un total de siete estudios que abarcó un total de 772 pacientes en el grupo ATC vs 756 pacientes del grupo de RAFI. Se observó menor tasa de mortalidad a cinco años en ATC vs RAFI (45.2\% vs $49.3 \%$; $R R=0.82$; IC 95\%, 0.68-0.98). La heterogeneidad obtenida fue de $\mathrm{I}^{2}=65 \%(\mathrm{p}<0.05)$, por lo que se realizó análisis por métodos aleatorios $(\mathrm{Fi}$ gura 2). Se hizo un análisis de funnel plot, donde se identificó un sesgo de publicación de dos estudios que confieren mayor riesgo de mortalidad al grupo de ATC, el resto de los estudios se encuentran dentro del intervalo de confianza y conservan la simetría entre ellos (Figura 3).

\section{DISCUSIÓN}

Los beneficios de la artroplastia de cadera se han descrito ampliamente y las indicaciones se han extendido a pacientes cada vez con menor edad, así como su papel en el tratamiento de las fracturas de cadera.

Por otro lado, la osteosíntesis de cadera ha mostrado tener ventajas como menor cantidad de sangrado o menos tiempo quirúrgico. ${ }^{5}$

Sin embargo, las complicaciones asociadas con osteosíntesis en fracturas de cadera ${ }^{6,7}$ pueden llevar a reoperaciones de los pacientes y requerir la colocación final de una prótesis de cadera; en la serie de Rodén, ${ }^{8}$ de 53 pacientes tratados con RAFI se requirió un total de 34 operaciones adicionales, de las cuales 12 fueron artroplastias como tratamiento final.

Los beneficios de cada uno de los métodos deben tomarse en cuenta e individualizarse antes de ser considerados como tratamiento de los pacientes con fractura de cadera.

La gran variabilidad en las poblaciones de los artículos en la literatura hace difícil establecer un seguimiento, tomando en cuenta los reportes de la mortalidad en artroplastia de cadera con seguimiento a corto y mediano plazo se registra un aumento de $0.12 \%{ }^{9}$ en la mortalidad en los primeros 26 días después de una artroplastia total de cadera primaria y de $0.41 \%$ a los 90 días de postoperados. ${ }^{10} \mathrm{Un}$ reporte a 10 años calcula una mortalidad acumulada de $15 \%,{ }^{9}$ que puede no estar asociada al tratamiento quirúrgico y se relaciona con la evolución de las enfermedades crónicas de los pacientes.

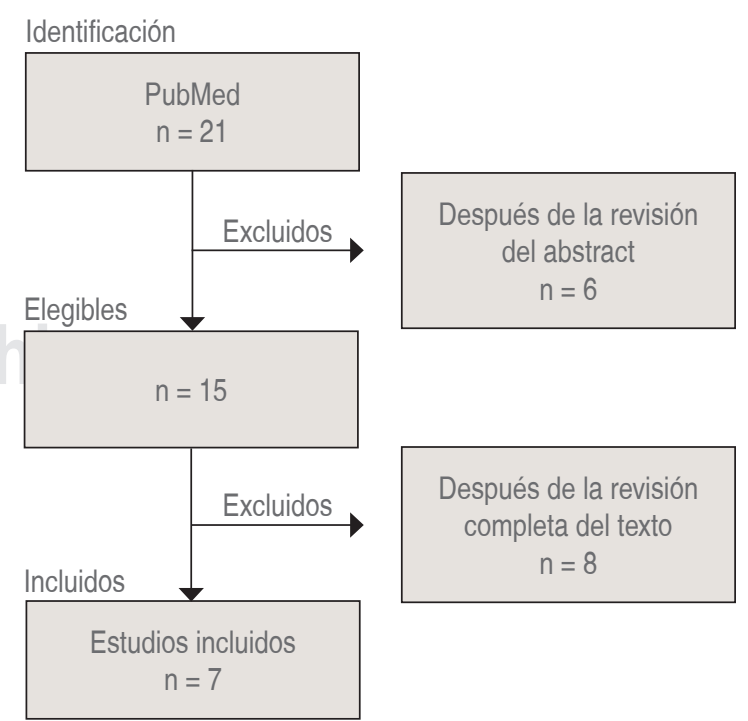

Figura 1: Diagrama de flujo de la selección de los estudios. 


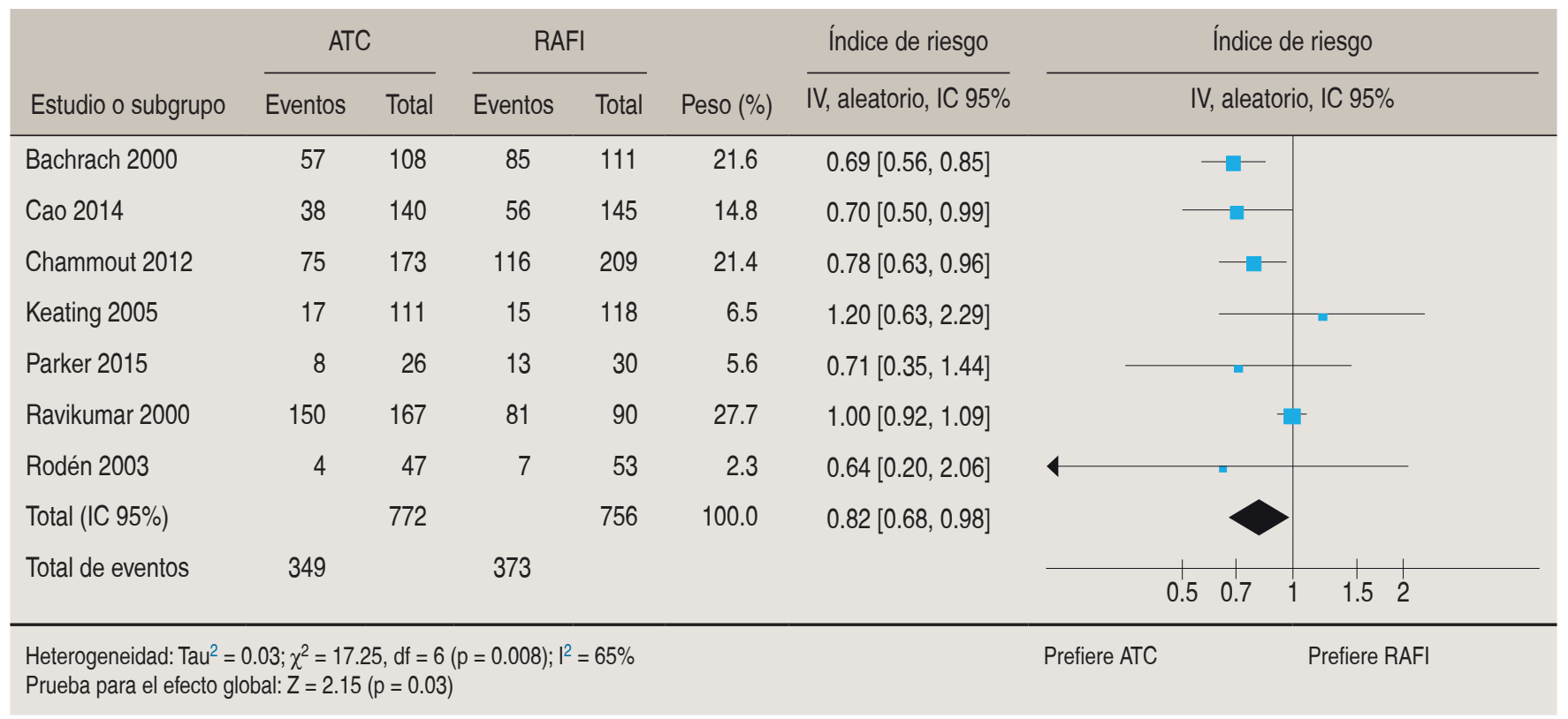

Acotaciones del riesgo de sesgo
(A) Generación de secuencia aleatoria (sesgo por la selección)
(B) Ocultación de la asignación (sesgo por la selección)
(C) Doble ciego, participantes y personal (sesgo por desempeño)

(D) Evaluación de resultados ciego (sesgo por detección)

(E) Resultados incompletos (sesgo por deserción)

(F) Reportes selectivos (sesgo por reportes)

(G) Otros sesgos

Figura 2: Forest plot: mortalidad a cinco años, artroplastia total de cadera vs reducción abierta y fijación interna. $\mathrm{RAFI}=$ reducción abierta y fijación interna; ATC = artroplastia total de cadera.

En cuanto a las características de los estudios incluidos en este metaanálisis encontramos que los pacientes presentaban un rango de edad de 75 a 84 años, cuatro estudios se llevaron a cabo en centros especializados en reemplazos articulares, un estudio fue multicéntrico y dos estudios no especifican el tipo de centro donde se realizaron. En cinco estudios se comparó la osteosíntesis con la colocación de tornillos canulados vs artroplastia y en dos estudios se comparó la osteosíntesis con placa vs artroplastia. En relación con las comorbilidades preoperatorias al comparar la osteosíntesis con artroplastia, sólo un artículo mostró mayor prevalencia de hipertensión arterial (HA) y enfermedad pulmonar obstructiva crónica (EPOC) en el grupo de osteosíntesis con una $\mathrm{p}=0.111$ y las comorbilidades previas no fueron tomadas como criterio para la asignación de la forma de tratamiento (ostesíntesis vs artroplastia).

Este metaanálisis tiene las siguientes fortalezas: Primero, aborda el estudio de un grupo de pacientes específico: pacientes con fractura de cadera. Segundo, compara directamente la mortalidad entre dos métodos de tratamiento. Tercero, establece un análi- sis en el tiempo postoperatorio poco estudiado: mortalidad a cinco años.

Una debilidad de este análisis es el número reducido de pacientes; sin embargo, establece una diferencia significativa en la tasa de mortalidad a cinco años.

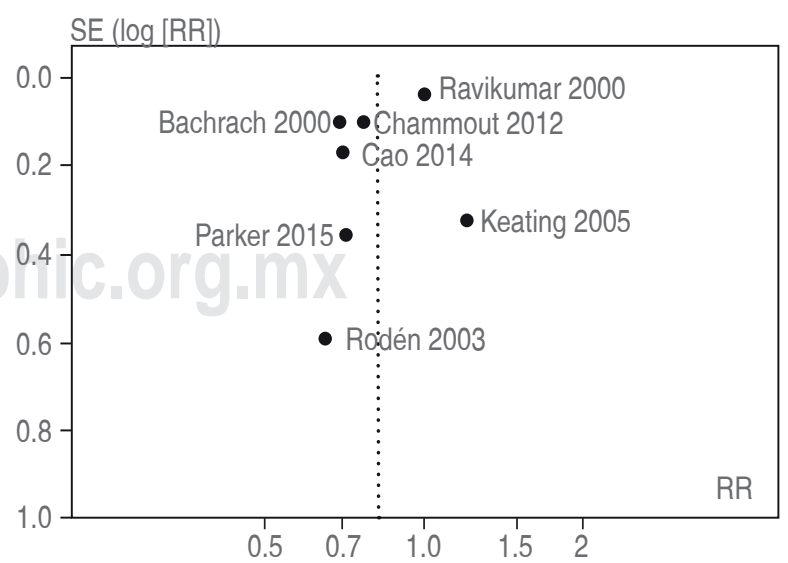

Figura 3: Funnel plot: sesgo de publicación de mortalidad a cinco años, artroplastia total de cadera vs reducción abierta y fijación interna funnel plot. $\mathrm{SE}=$ error estándar; $\mathrm{RR}=$ riesgo relativo. 


\section{CONCLUSIONES}

La ATC presenta menor tasa de mortalidad a cinco años vs fijación interna en pacientes de edad avanzada con fractura desplazada del cuello femoral.

La diferencia en la mortalidad en artroplastia de cadera vs fijación interna en los pacientes con fractura de cadera puede ser un elemento a considerar por el cirujano en la selección del método de tratamiento de los pacientes de edad avanzada con fractura de cadera cuando se tienen las dos opciones de tratamiento. Sin duda otro factor que debe influir en la toma de decisiones y desenlace del tratamiento es la experiencia del cirujano en reemplazos articulares.

\section{REFERENCIAS}

1. Bachrach-Lindstrom M, Johansson T, Unosson M, Ek AC, Wahlstrom O. Nutritional status and functional capacity after femoral neck fractures: a prospective randomized one-year follow-up study. Aging (Milano). 2000; 12 (5): 366-374.

2. Cao L, Wang B, Li M, Song S, Weng W, Li H et al. Closed reduction and internal fixation versus total hip arthroplasty for displaced femoral neck fracture. Chin J Traumatol. 2014; 17 (2): 63-68.

3. Chammout GK, Mukka SS, Carlsson T, Neander GF, Stark AW, Skoldenberg OG. Total hip replacement versus open reduction and internal fixation of displaced femoral neck fractures: a randomized long-term follow-up study. J Bone Joint Surg Am. 2012; 94 (21): 1921-1928.

4. Keating JF, Grant A, Masson M, Scott NW, Forbes JF. Displaced intracapsular hip fractures in fit, older people: a randomised comparison of reduction and fixation, bipolar hemiarthroplasty and total hip arthroplasty. Health Technol Assess. 2005; 9 (41): iii-iv, ix-x, 1-65.

5. Kim SY, Kim YG, Hwang JK. Cementless calcar-replacement hemiarthroplasty compared with intramedullary fixation of unstable intertrochanteric fractures. A prospective, randomized study. J Bone Joint Surg Am. 2005; 87 (10): 2186-2192.

6. Parker MJ. Hemiarthroplasty versus internal fixation for displaced intracapsular fractures of the hip in elderly men: a pilot randomised trial. Bone Joint J. 2015; 97-B (7): 992-996.

7. Ravikumar KJ, Marsh G. Internal fixation versus hemiarthroplasty versus total hip arthroplasty for displaced subcapital fractures of femur--13 year results of a prospective randomised study. Injury. 2000; 31 (10): 793-797.

8. Rodén M, Schon M, Fredin H. Treatment of displaced femoral neck fractures: a randomized minimum 5-year follow-up study of screws and bipolar hemiprostheses in 100 patients. Acta Orthop Scand. 2003; 74 (1): 42-44.

9. Dale H, Børsheim S, Kristensen TB, Fenstad AM, Gjertsen JE, Hallan G et al. Perioperative, short-, and long-term mortality related to fixation in primary total hip arthroplasty: a study on 79,557 patients in the -Norwegian Arthroplasty Register. Acta Orthop. 2020; 91 (2): 152-158.

10. Berstock JR, Beswick AD, Lenguerrand E, Whitehouse MR, Blom AW. Mortality after total hip replacement surgery: a systematic review. Bone Joint Res. 2014; 3 (6): 175-182. 